AVISO

Por no disponer de los correspondientes originales informáticos, la maquetación de este artículo difiere de la del publicado en papel. Por lo demás, los contenidos no han sufrido ninguna alteración.

Artículo publicado en el fascículo $2^{\circ}$ del tomo LXV (1997) de EMERITA, pp. 287-302

Autor: Idoia Mamolar Sánchez

\title{
LOS ANUNCIOS DEL CORO EN LA COMEDIA DE ARISTOFANES*
}

The entrances and exits of characters in Aristophanes' plays have been almost completely neglected by modern scholarship. This article is devoted to one particular element related to this aspect of dramatic technique: the entrance announcement. It do not study all announcements, but centre on those which are made by the chorus. These announcements are rare in Aristophanes: only 9 entrances are announced by the chorus, while 59 are announced by characters. The authoress selects the announcements by the former and study their form (metre, length, language), their content and the parts of comedy in which they occur, in order to assess the way in which Aristophanes uses them.

1. El movimiento escénico de entradas y salidas de personajes ${ }^{1}$ ha despertado escaso interés entre los estudiosos modernos de la comedia de Aristófanes ${ }^{2}$, y ello a pesar de que este aspecto de la técnica dramática del poeta está sujeto, como los demás, a las convenciones del género, siendo su estudio, en último término, un estudio de los parámetros de definición de la comedia anti-

* Este artículo ha sido elaborado dentro del proyecto de investigación Estudios sobre teatro griego: sistema y diacronía II (UPV/EHU 106.130-HA163/94), financiado por la Universidad del País Vasco. - Salvo que se indique lo contrario, para las citas y las referencias de número de verso se ha utilizado la edición de V. Coulon (trad. p. H. Van Daele), I-V, Paris 1923-30 (reed. post.).

1 El concepto de personaje engloba al personaje individualizado y al coro, poseedor al igual que aquél de una identidad como dramatis persona -más marcada en su caso en las escenas que preceden a la parabasis-. No obstante, con el fin de evitar confusiones, cuando sea preciso diferenciar entre uno y otro, reservaremos el término personaje para el personaje individualizado.

2 No así en el ámbito de la tragedia, donde en los últimos años está resultando una línea de investigación muy fructífera. Muestra de ello son los trabajos de O. Taplin, The Stagecraft of Aeschylus. The Dramatic Use of Exits and Entrances in Greek Tragedy, Oxford, 1977, D. Seale, Vision and Stagecraft in Sophocles, London-Canberra, 1982, y M. R. Halleran, Stagecraft in Euripides, London-Sydney, 1985, deudores éstos en gran medida del primero. Destaca también la monografía de E. García Novo, La entrada de los personajes y su anuncio en la tragedia griega, Madrid, 1981, donde puede verse un breve repaso de los autores que, con anterioridad a la misma, se han ocupado más o menos en extenso de la cuestión (pp. 15-19). El interés ha llegado también a la comedia de Menandro (K. B. Frost, Exits and Entrances in Menander, Oxford, 1988). 
gua (al menos, de la aristofánica). En efecto, prescindiendo de las referencias hechas al hilo del análisis formal de los elementos compositivos de la comedia -en especial de la parodos (así, en su reciente monografia B. Zimmermann $^{3}$ se ocupa de las cuestiones formales y de otras como la motivación y preparación de la entrada del coro, y la relación de éste con la acción dramática)-, una de las líneas de investigación más sólidas y consolidadas, la atención prestada a las entradas y salidas de personajes se reduce a breves apuntes que aparecen de forma esporádica en algunas ediciones comentadas y ciertos trabajos, como el de C. F. Russo ${ }^{4}$, muy interesado por el componente teatral de las comedias -tradicionalmente estudiadas como obras literarias-, o el de J. Andrieu ${ }^{5}$ sobre el diálogo antiguo, citados ambos porque contienen algunas observaciones acerca del elemento relacionado con el movimiento escénico que vamos a tratar aquí: el anuncio de entrada en escena.

Centrándonos ya en el anuncio, si nos remontamos a fechas bastante anteriores, hallamos un estudio en el que se abordan con cierto detalle distintos aspectos del mismo. Nos referimos al trabajo publicado en 1893 por E. Bodensteiner ${ }^{6}$, que aporta datos sobre el número de anuncios por obra, los formulados por los personajes y por el coro, el metro empleado o la extensión de los mismos, entre otros (contiene, además, un extenso apéndice con la relación de entradas y salidas de todas las comedias, 759-806). También merece ser destacado el de W. Koch ${ }^{7}$, un poco posterior, en el que se estudia el carácter «formular» del anuncio en las comedias griega y latina, así como el de otros elementos ligados a los movimientos escénicos; la advertencia que hace uno mismo de su propia salida, la llamada con la que se solicita la presencia de un personaje ausente -preparando así su aparición- o la intervención del recién llegado son algunos de ellos.

Volviendo al primero, cuando Bodensteiner da las cifras de los anuncios en boca del coro y de los que están en boca de los personajes, llama la aten-

\footnotetext{
3 Untersuchungen zur Form und dramatischen Technik der Aristophanischen Komödien. Parodos und Amoibaion I, Königstein/Ts., 1984.

$4 \quad$ Aristofane autore di teatro, Firenze $1984^{2}$ (ampl. e agg.) $\left(1962^{1}\right)$.

5 Le dialogue antique: structure et présentation, Paris, 1954, esp. pp. 196-199.

$6 \quad$ Szenische Fragen über den Ort des Auftretens und Abgehens von Schauspielern und Chor im griechischen Drama, Jb. f. cl. Phil. Suppl. Bd. XIX, 1893, pp. 637-808, esp. pp. 705 ss.

7 De personarum comicarum introductione, Diss., Breslau, 1914.
}

EMERITA. Revista de Lingüística y Filología Clásica (EM) - LXV 2, 1997, pp. 287-302 
ción sobre el reducido número de aquéllos ( 7 frente a 36 , según los datos del autor $)^{8}$, un hecho que ha sido señalado también en fechas más recientes por Russo, aunque sin ofrecer en su caso ningún dato sobre los anuncios formulados por los personajes; Russo (Aristofane, p. 116) declara sin más lo siguiente: «In tutto Aristofane il Coro annuncia l'arrivo dei personaggi in neanche una diecina di cassi.» Bodensteiner, por otra parte, compara la situación con la de la tragedia, donde nos encontramos con un panorama del todo distinto. En efecto, en la tragedia las entradas anunciadas por el coro son en términos absolutos considerablemente más numerosas que las anunciadas por los personajes, y aunque es cierto que estos últimos fueron ganándole terreno a aquél paulatinamente, nunca se llegó a una situación ni siquiera parecida a la de la comedia: los anuncios del coro y los formulados por los personajes tendieron a igualarse ${ }^{9}$. Pues bien, la escasa presencia de los anuncios del coro en Aristófanes, y que ambos autores se limitan prácticamente a constatar (resulta evidente en el caso de Russo), es lo que ha hecho que nos parezca interesante centrarnos en su estudio, que ya hemos llevado a cabo en

$8 \quad$ Szenische Fragen 706. Como veremos, estas cifras (Bodensteiner no menciona expresamente los anuncios) no coinciden con las nuestras, especialmente por lo que se refiere al número de anuncios formulados por los personajes, que es, según el concepto de anuncio que nosotros utilizamos y que determinaremos a continuación, más elevado que el dado por el autor (59); en cuanto a los del coro, hay dos más (9). Bodensteiner reserva el término de anuncio para las introducciones más típicas, como «alguien viene»o «aquí está éste», con las que se constata de forma explícita una entrada; según esta definición, p. ej., Aves 1196-8, donde el corifeo sólo señala que se oye la aproximación del personaje, no sería para él un anuncio, pero sí para nosotros. Siguiendo con las introducciones en boca del coro, el hecho de que Cab. 1326-1332 sea un anuncio dialogado entre Morcillero y el corifeo, en el que la constatación de la entrada de Pueblo recae en el personaje de Morcillero, permite no incluirlo dentro de los anuncios formulados por el coro; si suprimimos también éste (lo que nosotros no hemos hecho), se llega a la cifra de 7 dada por Bodensteiner. El recuento de los anuncios a cargo de los personajes resulta más problemático; aun contabilizando exclusivamente los anuncios del tipo más estereotipado, el número de éstos ronda los 50 , una cifra muy alejada todavía de la ofrecida por el autor. En fin, según nuestros datos la diferencia entre el número de introducciones formuladas por el coro y aquellas en boca de los personajes es aún mayor.

9 Taplin, Stagecraft, p. 71, apunta el cambio habido de Esquilo a Eurípides; en el primero la mayor parte de los anuncios están en boca del coro. Para los datos precisos, véanse G. Novo, Anuncio, pp. 564-565, y Bodensteiner, Szenische Fragen, pp. 705-706, cuyas cifras para la tragedia, a la vista de las ofrecidas por G. Novo, parecen más fidedignas que las dadas para la comedia (piénsese sobre todo en las referentes al número de anuncios pronunciados por los personajes).

EMERITA. Revista de Lingüística y Filología Clásica (EM) - LXV 2, 1997, pp. 287-302 
otro lugar para los anuncios de las cinco comedias más antiguas ${ }^{10}$; ahora nos proponemos recoger los existentes en las otras seis y analizar su forma, su distribución y contenido, lo que nos permitirá elaborar conclusiones definitivas sobre el empleo que el poeta cómico hace de ellos.

Antes de emprender esta tarea es necesario aclarar qué entendemos por anuncio. El término lo empleamos según la definición dada por Taplin (Stagecraft, p. $71 \mathrm{s.}$ ):

A working definition might be 'anything said about the approach of a character after that approach has first been seen (or otherwise perceived) by someone already on stage' - Entrance announcements are a phenomenon of the open stage - Since considerable distances had to be traversed by characters joining or leaving the play, the lines of announcement doubtless helped to cover and accompany these long movements. But this observation by no means accounts exclusively for the convention. Many entries from the skene door, which would not take so long, are nevertheless announced, while many entrances from the side eisodoi are not announced at all.

Sobre el hecho de que la entrada sea "percibida de otra manera», Taplin amplía en una nota (p. 71, n. 3) esta afirmación, señalando la posibilidad de que sean el ruido o el olor lo que advierta a los presentes en escena de la llegada de otro personaje. Así pues, en opinión del autor, el anuncio no ha de ser exclusivamente de naturaleza visual.

10 Como parte del trabajo de investigación de la tesis doctoral que estamos realizando. Titulada Los movimientos escénicos en la comedia de Aristófanes. Un estudio de técnica teatral, en ella nos ocupamos de este primer grupo de obras, con unas características propias dentro de la producción de Aristófanes, como es comúnmente admitido. La mejor visión de conjunto sobre las distintas etapas de la obra de Aristófanes nos parece la de Th. Gelzer, Der epirrhematische Agon bei Aristophanes, München, 1960, pp. 240-276. Partiendo de un criterio cronológico, establece la habitual clasificación en tres periodos: el primero lo constituyen las cinco comedias más antiguas, que van del año 425 -fecha de Acarnienses, la primera conservada- al 421 -fecha de la representación de $P a z-;$ el segundo comprende las piezas representadas entre los años 414 y 405 -Aves, Lisistrata, Tesmoforiantes y Ranas-; y el tercero, las dos últimas comedias llegadas a nosotros, pertenecientes a los primeros años del s. IV, Asambleistas -representada probablemente en 392- y Pluto -388-. Dentro de cada periodo, Gelzer estudia los cambios de forma, función y contenido que experimentan progresivamente los elementos compositivos tradicionales (en especial el agon, la parabasis, las escenas yámbicas que siguen a ésta y las partes cantadas), así como las transformaciones habidas en la organización de la acción dramática, en el contenido de las obras y en los tipos de personajes, todo lo cual hace que cada una de las tres etapas, por encima de lo que la une a las demás, constituya algo diferente.

EMERITA. Revista de Lingüística y Filología Clásica (EM) - LXV 2, 1997, pp. 287-302 
Muy diferente es el concepto utilizado por G. Novo (Anuncio). Entendiéndolo en un sentido más amplio, distingue los tipos de «anuncio inmediato» $\mathrm{y}$ «mediato», estableciendo a su vez una variedad de modalidades dentro de cada uno de ellos (cf. esp. pp. 34-5). A propósito de estas modalidades, queremos destacar, por un lado, que incluyen diversas formas de lo que Taplin considera «preparación» (Stagecraft 9 s.: « «anything said or done before an entry or exit which prepares for that event or has a bearing on it'»), y que nosotros, como él, distinguimos claramente del anuncio de entrada en escena; y, por otro, que la modalidad de «anuncio inmediato» denominada «anuncio explícito» (entendiendo por tal «exclusivamente la declaración de que un personaje llega a escena, hecha por el personaje que lo divisa», Anuncio 222) es la que coincide básicamente con nuestro concepto de anuncio, si bien éste es más amplio, como lo reconoce la propia G. Novo (41). En efecto, al examinar la definición de Taplin, concluye que dicha definición comprende otros dos subtipos además del de «anuncio explícito», los de «Voz en off sin palabras» $y$ «¿Es él?». En el primero tienen cabida los comentarios que provocan en los presentes en escena los ruidos o pasos que alertan de la llegada de un personaje, y en el segundo, las dudas expresadas por aquéllos sobre la identidad del personaje que se aproxima (Anuncio 37). Para nosotros, insistimos, sólo hay un «anuncio» de entrada en escena y estas tres modalidades están incluidas dentro del mismo.

2. En el conjunto de comedias que se abre con Aves y termina con la última de las que han llegado hasta nosotros, Pluto, encontramos únicamente cinco anuncios del corifeo, cuya extensión oscila entre los dos versos de Lis. 10723 y los cuatro de Lis. 1082-5 y As. 500-3; los dos restantes son grupos de tres versos (Aves 1196-8 y Tesm. 571-3). Por lo que se refiere al metro, hallamos una cierta variedad: trímetros yámbicos (Aves 1196 ss. ${ }^{11}$ y Lis. 1082 ss.), te

11 En el caso del primer verso, los mss. nos han transmitido un dímetro yámbico. Para las propuestas que se han hecho con el fin de restaurar el trímetro esperado, cf. el aparato crítico de las ediciones de J. van Leeuwen, Aves, Leiden, 1968 (=1903), quien corrige el verso, y O. Schroeder, Die Vögel, Berlin, $1927^{4}$ (ganz neue Bearb. von Th. Kock, Leipzig, 1895 ${ }^{3}$ ), quien no lo hace pero supone la pérdida de parte del mismo, como N. Dunbar, Birds, Oxford, 1995, en su reciente edición de la obra (uid. ad 1196). Coulon enmienda el verso. Por su parte, A. H. Sommerstein, Birds, Warminster, 1987, ad loc., señala la existencia ocasional de irregularidades como ésta en el verso yámbico, no creyendo necesaria su corrección; por lo que se refiere a este caso, apunta la posibilidad de que el dímetro se relacione con cuestiones 
trámetros yámbicos (Tesm. 571 ss. y As. 500 ss.) y, finalmente, tetrámetros anapésticos en el anuncio de Lis. 1072-3. Cabe señalar que salvo este último, que constituye desde el punto de vista métrico una secuencia aislada (entre un stasimon, 1043-71, y el recitado en trímetros, 1074 ss.), todos los demás forman parte de un conjunto más amplio en el mismo metro. Así, en una escena yámbica ${ }^{12}$ aparecen los anuncios de Aves 1196 ss. y Lis. 1082 ss., aquél al comienzo de la unidad (1196-261) y éste en el interior de la misma (1074107); Tesm. 571 ss. son los tres versos finales de la escena en tetrámetros iniciada en 531; por último, As. 500 ss. es el antepirrema de la sizigía que constituye la epiparodos (483-503) ${ }^{13}$, sizigía cuyo epirrema (489-92) está en tetrámetros yámbicos, encontrándose también este metro en las partes líricas (483-8=493-9), junto a dímetros yámbicos, y en los versos introductorios del conjunto $(478-82)^{14}$.

Respecto a la posición en que aparecen, si exceptuamos Lis. 1082-5, el resto sirven de transición de un elemento coral (stasimon y sizigía en el caso de los anuncios de Lis. 1072-3 y de As., según acabamos de ver, y estrofa en el de Aves-1188-95, en responsión con 1262-8-) o de una escena en versos largos básicamente a cargo de los personajes, como sucede en Tesm. (los vv. 531-2 y 571-3 constituyen las únicas intervenciones de la corifeo, aunque el coro es apelado continuamente por el Pariente de Eurípides y la Mujer $1^{\mathrm{a}}$, de tal manera que, a pesar de su silencio, se hace notar su presencia), a una es-

de acompañamiento musical.

12 Entendemos este concepto según un criterio métrico. Así, escena yámbica es toda parte recitada en trímetros a cargo de los personajes de la skene -aunque ocasionalmente puede incluir otro tipo de versos, también líricos, y breves intervenciones del corifeo-, con independencia del lugar en que aparezca (ya sea el característico, después de la parabasis -cuando la hay-, o antes de la misma) o de los elementos formales que le sirvan de marco (canto coral

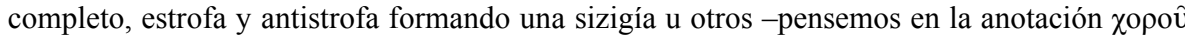
que aparece en los manuscritos de las dos últimas obras-).

13 La propuesta de considerar estos versos una sizigía en vez de una canción antistrófica fue de P. Mazon (Essai sur la composition des comédies d' Aristophane, Diss. Paris 1904, 153 , n. 4), a quien sigue F. Perusino (Il tetrametro giambico catalettico nella commedia greca, Roma 1968, 38 s.); Zimmermann, Form u. Technik I 138, por su parte, opina que es plausible. Van Daele se inclina por la otra opción, englobándolos en el canto.

14 Tetrámetros yámbicos se encuentran igualmente en los cuatro versos con los que la corifea exhorta a sus compañeras a acudir a la Asamblea (285-8), abriendo así la intervención con la que el coro abandona la orquestra tras el prólogo. Sobre la función caracterizadora del metro en las parodoi de las comedias de Aristófanes, Zimmermann, Form u. Technik I $146 \mathrm{~s}$. 
cena yámbica. En definitiva, estas introducciones en boca del corifeo cumplen una función de puente, aminorando el hiato que existe entre dos unidades de carácter distinto.

Siendo necesariamente los anuncios corales anuncios de la entrada de los personajes individualizados, constituyen por sí mismos una manera particular de contacto del coro con la skene, y ello con independencia de la forma que adopten, el lugar en el que aparezcan o su posición en el mismo, aspectos que acaban de ser analizados. Pues bien, como vamos a ver ahora, salvo en Aves, ese contacto prosigue tras la introducción del personaje, lo que significa, teniendo en cuenta lo dicho acerca de la distribución de los anuncios, una integración del coro en las escenas yámbicas que merece ser destacada ${ }^{15}$. En todos los casos se produce un diálogo corifeo-personaje, iniciado normalmente por este último. Así, en As., cuando Praxágora regresa de la Asamblea, establece contacto inmediato con las mujeres del coro mediante un

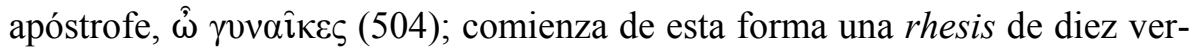
sos en la que recuerda a sus compañeras el éxito logrado en la junta (505), las apremia para que se quiten el disfraz de varones que llevan puesto (506$10 \mathrm{a})^{16} \mathrm{y}$, por último, les comunica su intención de introducirse en su propia

15 Por lo que se refiere al anuncio de Aves, el hecho de que se encuentre en una escena yámbica supone por sí mismo una integración del coro en esta parte estructural de la comedia, reservada propiamente para los personajes de la skene. De otro lado, es cierto que el corifeo enmudece tras el anuncio, a diferencia de lo que sucede en los casos restantes, pero al menos temáticamente sigue ligado a lo que acontece sobre el escenario, y no es descartable que sea él el destinatario de la pregunta formulada por Pistetero en 1211 (cf. Dunbar, ad loc.). Con todo, ninguna de las dos circunstancias puede compararse con la participación verbal que tiene el corifeo en los demás casos.

16 Seguimos la opinión de R. G. Ussher, Ecclesiazusae, Oxford, 1973, ad 500-2 y 50913, al considerar que la protagonista entra sola en escena y que, por tanto, el coro es el único destinatario posible de todas las apelaciones; ad 500-2: «The chorus, of course, have eyes only for the general (en el anuncio, según la propuesta de Ussher para 503, sólo se menciona a Praxágora): but it seems clear she is entering alone. We hear no more of Women $A$ and $B_{-}$, with whom she had gone off (279).» No obstante, su lectura del último verso del anuncio (cf. ad 503) no descarta que Praxágora esté acompañada (independientemente, como hemos señalado, de que luego el autor no encuentre apoyo en el texto para creerlo así): $\chi \alpha \hat{\tau} \tau \alpha \imath \gamma \grave{\alpha} \rho$

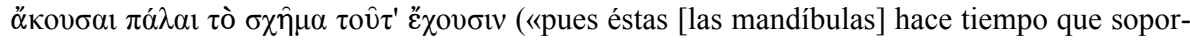
tan este disfraz [la barba postiza] sin quererlo»). La lectura de Coulon, en cambio, que es la de los mss., implica claramente que la protagonista regresa de la Asamblea con otras mujeres

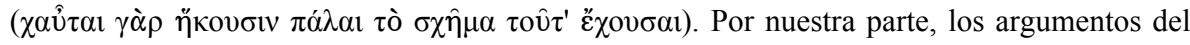


casa antes de que su marido la vea (510b-3). Tienen lugar a continuación sendas intervenciones en versos largos ${ }^{17}$ (tetrámetros anapésticos) de la corifeo y de la protagonista (514-6 y 517-9): la primera se dirige a Praxágora para decirle que su orden ya ha sido cumplida y que están dispuestas a seguir obedeciéndola, y la segunda recoge el ofrecimiento, pidiéndoles que aguarden y actúen como sus consejeras en el momento oportuno. En los demás casos, el diálogo es en trímetros yámbicos, dándose la circunstancia de que las intervenciones del corifeo en estos pasajes se encuentran entre las más extensas de las suyas en dicho metro ${ }^{18}$. Se trata, de un lado, del conjunto de Lis. 1074-95 (doce versos del corifeo, incluido el anuncio de 1082-5), que comprende los dos diálogos sucesivos que mantiene el corifeo con los embajadores de Esparta (1074-81) y Atenas (1086-95), iniciado el primero por él mismo (saluda a los laconios y les interroga sobre su estado -recordemos que aparecen con una erección, fruto de la abstinencia sexual a la que les tienen sometidos sus mujeres, persuadidas por Lisístrata-, 1074-5) y el siguiente por el personaje que efectúa su entrada (el portavoz de los atenienses, aquejados de la misma enfermedad que los lacedemonios y deseosos como ellos de que la protagonista les reconcilie y recuperen así a sus mujeres, pregunta por el paradero de Lisístrata $\left.{ }^{19}, 1086-7\right)$; y, de otro lado, de Tesm. 574-602, un diálogo, esencialmente de la corifeo (diez trímetros) con Clístenes $^{20}$, que constituye un caso especial. Su peculiaridad: es una parodia

primero sobre la necesidad de enmendar el verso y la justificación de la lectura propuesta por él nos parecen razonables para aceptar ésta. Respecto al parlamento de Praxágora, Ussher (ad 504-13) observa un cierto colorido trágico en el estilo y en el metro.

17 Los trímetros yámbicos (520-70) reaparecen tras este pequeño grupo de tetrámetros, pero pertenecen por entero a los personajes. Sobre esta combinación de trímetros y versos largos, Th. Gelzer, «Aristophanes der Komiker», RE Suppl.-Bd. 12, 1971, cols. 1391-570, $1504-5$ y 1521.

18 W. Schmid-O. Stählin, Geschichte der griechischen Literatur I, 4, München, 1946, 58, n. 7.

19 La pregunta no tiene un destinatario explícito, pero es el corifeo quien toma la palabra tras ella con un comentario acerca de la erección que padecen también los atenienses y con una cuestión formulada expresamente a éstos (1088 s.). Por lo que sabemos, sólo tras el diálogo con el corifeo el embajador ateniense se percata de la presencia de los espartanos, dirigiéndose a ellos mediante un saludo (1097); los espartanos no han abandonado la escena desde su entrada en el v. 1072.

${ }_{20}$ Para ser rigurosos, hay que hacer algunas matizaciones al respecto. Así, Clístenes, al entrar, no se dirige expresante a la corifeo sino, en general, a las mujeres presentes en escena, 
de uno de los componentes del esquema más estereotipado que adopta la intervención del mensajero en la tragedia, sobre todo de la mano de Eurípides, el diálogo de entrada; este diálogo sirve de introducción a la rhesis en la que el personaje relata detalladamente la noticia que le ha traído a escena, rhesis que falta en nuestro caso. Clístenes, en efecto, cumple la función de mensajero que revela a las mujeres el plan urdido y llevado a cabo por Eurípides para evitar el castigo que éstas desean imponerle por difamarlas en sus tragedias: Eurípides ha introducido entre ellas a un pariente suyo disfrazado de mujer para espiarlas. Por otra parte, la parodia, como veremos, abarca también al propio anuncio, otro de los componentes de la escena de mensaje$\mathrm{ro}^{21}$.

En cuanto al diálogo de entrada, la parodia del mismo determina la estructura y, en buena medida, el contenido de Tesm. 574-602. Elementos típicos de este diálogo en la tragedia son el apóstrofe con el que el mensajero se dirige a su interlocutor $(574)^{22}$ y los comentarios de aquél acerca de la noticia que trae, comentarios que ponen de manifiesto el carácter negativo de la misma en este caso (Clístenes se refiere a ella como $\pi \rho \hat{\alpha} \gamma \mu \alpha \pi \varepsilon \rho \grave{i} \dot{v} \mu \hat{\omega} v$

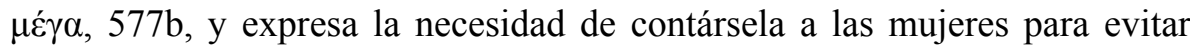

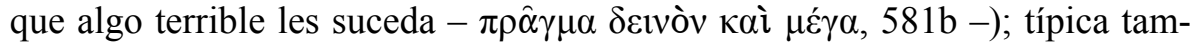

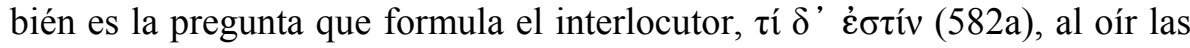
palabras anteriores del mensajero; y lo son igualmente el comunicado escue-

esto es, al coro, al Pariente -no descubierta todavía su verdadera identidad-y a la Mujer $1^{\text {a }}$; no obstante, es la corifeo quien interviene a continuación, iniciando un intercambio de réplicas con el recién llegado que finaliza en 591. El diálogo se diversifica en 592, cuando el Pariente toma la palabra para hacer a sus compañeras un comentario irónico sobre lo dicho por Clístenes (592-4), y a su vez éste se dirige a él reprochándole su actitud (595-6). Los interlocutores vuelven a ser la corifeo y Clístenes en 601-2, no sin que antes aquélla haya apelado a las demás mujeres (597-600). Finaliza así el diálogo de entrada, pero las intervenciones de la corifeo en trímetros se repiten en 607 y 613-4.

${ }^{21}$ Aunque la escena de mensajero alcanza un grado de formalización notable, especialmente, como hemos señalado, de la mano de Eurípides, no sigue un esquema rígido; así, por ejemplo, el anuncio es un elemento aleatorio de la misma. Véase el análisis de P. Rau, Paratragodia, München, 1967, pp. 162-168, de la parodia que hace Aristófanes en sus comedias de la escena de mensajero (para este pasaje concreto, $46 \mathrm{~s}$.).

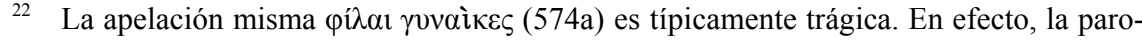
dia no sólo está presente en la adopción de un esquema formal sino también en los aspectos verbal, de estilo y métrico. Nosotros, no obstante, vamos a referirnos básicamente a lo primero; para la otra cuestión remitimos al análisis ya señalado de Rau. 
to de la noticia por parte de éste (584 s.) y la breve serie de preguntas-respuestas a cargo de la corifeo - interesada en ampliar la información que acaban de comunicarle - y de Clístenes (586-91). Finalmente, en cuanto a la forma del diálogo, la tendencia a la esticomitia que éste presenta en la tragedia la encontramos también en los vv. 582-91.

Respecto al otro elemento parodiado, la introducción del mensajero, su análisis será el punto de partida para estudiar el aspecto del que vamos a ocuparnos ahora: el contenido de los anuncios; y ello por una razón, la de que el contenido de Tesm. 571-3 está determinado en parte por la parodia misma.

Dos son, concretamente, los motivos que se toman prestados. En primer lugar, el de la prisa con la que el personaje se aproxima ( $\dot{\varepsilon} \sigma \pi 0 v \delta \alpha \kappa v i \hat{\alpha} \alpha$ $\pi \rho 0 \sigma \tau \rho \varepsilon ́ \chi \varepsilon 1,572 a)$, un motivo frecuente en los anuncios de mensajero, aunque no exclusivo de ellos; se trata de un recurso dramático que crea una sensación de premura y aviva la expectación que despierta por sí misma la entrada. De igual manera hay que entender el segundo, también ampliamente utilizado en la tragedia ${ }^{23}$ : se anticipa que el personaje trae noticias ( $\check{\tau} \tau \alpha$ $\lambda \varepsilon \dot{\varepsilon} \xi \varepsilon 1,573 b)$. En cuanto al resto del anuncio, contiene la reacción de la corifeo ante la entrada (ordena al Pariente y a la Mujer $1^{a}$ cesar en su disputa, 571a, y pide silencio para poder escuchar lo que va a decirse, 573) y la iden-

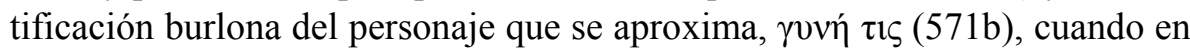
realidad se trata de Clístenes ${ }^{24}$.

23 Para el uso de ambos recursos por los poetas trágicos, Taplin, Stagecraft 147. En su análisis Rau sólo menciona el primero; tampoco dice nada sobre la petición de silencio de la corifeo (573), un motivo frecuente en los anuncios de la tragedia (G. Novo, Anuncio, p. 569).

24 Su naturaleza afeminada es uno de los elementos que rompen cómicamente el carácter estilizado de la intervención del mensajero en la tragedia (la noticia misma y la actitud irónica del Pariente, 592-4, contribuyen también a ello). Por otra parte, como lectores no sabemos con certeza que se trata de Clístenes hasta que en 634 (60 versos después de su entrada) se le llama por su nombre (es la única aparición del personaje y no ha sido preparada); en cambio, desde el primer momento se sabe que es un hombre, pues se expresa como tal, empleando formas masculinas para referirse a sí mismo (cf. esp. 575-9). S. D. Olson, «Names and Naming in Aristophanic Comedy», $C Q 42,1992,304-19,316-8$, señala que la mayoría de los personajes que aparecen sobre la escena cómica encarnando a individuos de carne y hueso son identificados explícitamente por el nombre justo antes de su entrada o inmediatamente después de ésta, como si el poeta tuviera poca confianza en que el público los reconociera sólo con verlos, o lo que es lo mismo, sólo por su apariencia y comportamiento (316-7). A propósito de Clístenes (318), apunta que debía de tener una fisonomía tan característica que 
Las demás introducciones son también algo más que la mera constatación de una entrada, ofreciendo todas ellas información acerca de al menos dos de los datos contenidos en el anuncio de Tesm.: la identidad del personaje que aparece en escena, ya sea por primera vez (los embajadores de Esparta y los de Atenas, Lis. 1072 y $1082^{25}$; un dios alado, sin especificar cuál, Aves 1197 s. -leyendo el texto, no sabemos que se trata de Iris hasta la mención de su nombre en $1204^{26}$-) o lo haya hecho anterior-

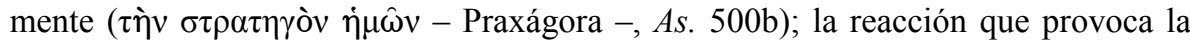
entrada (en Aves 1196, el corifeo exhorta a sus compañeros a estar vigilantes, y en As. 500-3, la corifea insta a las demás a quitarse deprisa sus disfraces de hombre); o circunstancias particulares de la entrada misma o de quien la efectúa (en los dos anuncios de Lis. se presta atención a un hecho visual llamativo, la erección con la que los dos grupos llegan a escena, 1073 y 1083-5; en el de los espartanos se alude además a otra característica de su aspecto, la barba larga $-1072 b-$, marca distintiva frente a la recortada al estilo ateniense ${ }^{27}$ ). En este sentido, el anuncio de Aves resulta un caso peculiar, ya que contiene la alusión a un contacto aural, previo a la aparición del personaje (crea la expectativa de ésta), que no se encuentra en ninguno de los otros, faltando, en cambio, el anuncio visual propiamente dicho; en efecto, el corifeo se limita a señalar que se oye cerca el ruido de las alas del dios (1197-8), dirigiéndose a continuación Pistetero a Iris (1199). Otra característica, además de la del contacto aural, diferencia al anuncio de Aves de los restantes: el estilo elevado de sus dos últimos versos ${ }^{28}$. Con una breve referencia a esta cuestión terminaremos nuestro aná

permitía su inmediata identificación por la máscara (señalado ya antes por K. J. Dover,

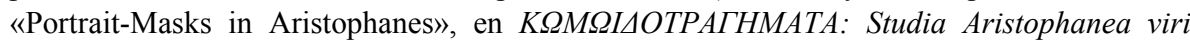
Aristophanei W. J. W. Koster in honorem, Amsterdam 1967, 16-28, 20 [= H.-J. Newiger (ed.), Aristophanes und die alte Komödie, Darmstadt 1975, 155-69, 159 s.]); se refiere, en concreto, a su condición de barbilampiño, blanco de las burlas de Aristófanes en varios pasajes de sus comedias, entre los que se encuentra también éste (Clístenes alude a sus mejillas despobladas al comienzo mismo de su intervención, 575; el motivo se repite en 583).

25 Seguimos a J. Henderson, Lysistrata, Oxford, 1987, al considerar que el portavoz de los atenienses no ha aparecido anteriormente en escena. Coulon otorga este papel al mismo personaje (Prítanis, en su denominación) que, según él, habría entrado en 982 y departido con el Heraldo laconio hasta 1013. Henderson (ad 980-1013) argumenta de forma convincente lo correcto de atribuir a Cinesias las intervenciones que Coulon pone en boca del supuesto Prítanis.

26 Olson, «Names and Naming», p. 316, considera probable que tampoco el espectador lo supiera con seguridad hasta entonces, ya que, según señala, Iris no parece haber tenido unos atributos particularmente distintivos que permitieran su inmediato reconocimiento; por otra parte, las alusiones a dioses alados, previas a la aparición del personaje, no hacen posible determinar de quién se trata (cf. 572-5 y 1170 ss.).

27 Henderson, ad 1072.

28 Vid. las anotaciones a estos versos hechas por Schroeder, van Leeuwen y Dunbar en

EMERITA. Revista de Lingüística y Filología Clásica (EM) - LXV 2, 1997, pp. 287-302 
lisis.

Destacan en ellos el adjetivo eólico $\pi \varepsilon \delta \alpha ́ \rho \sigma 10 \varsigma$ (1197b), de corte poético,

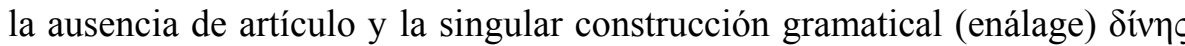

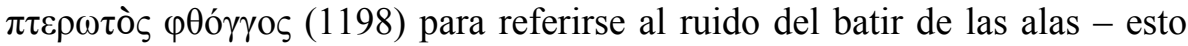

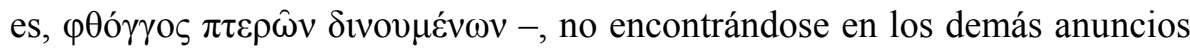
nada parecido ${ }^{29}$. El de éstos es un estilo prosaico (aunque el del mensajero sobresale por su carácter paródico). Se repiten en ellos, por otra parte, algunos elementos, lo que les da cierta uniformidad: las típicas partículas intro-

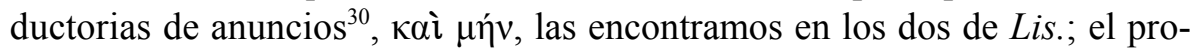
nombre demostrativo ő $\delta \varepsilon$, referido al personaje que entra en escena, aparece en todos salvo en el de Tesm.; se repiten los verbos ó $\hat{\alpha} v$ (Lis. 1082 y As.

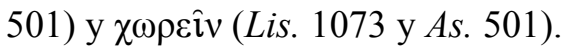

3. Llegados a este punto, no nos queda sino mirar atrás y poner uno al lado del otro el conjunto de anuncios que acabamos de analizar y el conjunto de los que encontramos anteriormente en las cinco primeras comedias: $A c$. 238-40 y 1069-70, Avisp. 1532-4 y Cab. 1326-32, un anuncio dialogado en el que interviene el corifeo aunque es el otro interlocutor (Morcillero) quien propiamente introduce al personaje que aparece en escena (Pueblo). Las preguntas que surgen son dos: 1) ¿Pueden formularse algunos principios básicos acerca del uso de los anuncios corales en la comedia de Aristófanes? 2) ¿Son los anuncios de las obras posteriores a $\mathrm{Paz}$ algo distinto de los de las obras precedentes? Como vamos a ver, la respuesta en ambos casos es afirmativa. Efectivamente, además del principio básico fundamental, que es la escasez misma de los anuncios del coro, hay otros - por encima de ciertas particularidades- que atañen a la forma y al contenido de éstos; respecto a la segunda pregunta, se observa una diferencia clara en lo concerniente a la distribución de los anuncios y al contacto posterior del coro con la skene.

sus respectivas ediciones de Aves.

29 Rau, Paratragodia, p. 197, señala, además, el ritmo trágico de los dos trímetros. Todo ello ha hecho pensar que ambos versos son, en realidad, la cita de alguna tragedia (adesp. 47 Nauck). Por otro lado, el género trágico está ya presente en la preparación de la entrada de Iris (recordemos la intervención paródica del mensajero que da la noticia de la infiltración de un dios en la ciudad de las aves, 1168 ss., y la reacción lírica del coro en docmios, 1188 ss.) y en parte de su intervención después de aparecer en escena (1231-3 y 1238 ss.), lo que cabe entenderse como una forma de caracterización de la diosa (Rau, ibíd., pp. 165 y 197 s.).

${ }^{30}$ Las hallamos en Aristófanes y en los poetas trágicos (sobre todo en Eurípides); cf. J. D. Denniston, The Greek Particles, Oxford, $1954^{2}$, pp. 356 y 586. 
Empecemos por las características comunes:

Los anuncios del coro son pocos, y la relación entre el número de éstos y el de los formulados por los personajes no varía de forma significativa en las distintas etapas de la producción del poeta cómico. Así, de Acarnienses a $P a z$ hay 4 anuncios del corifeo (representan un 13\% del número total) y 27 en boca de los personajes; de Aves a Ranas, 4 (casi un 15\%) y 23, respectivamente; y, por último, en Asambleístas y Pluto, 1 (el porcentaje más bajo, un $10 \%)$ y 9 . Se trata, por otra parte, de anuncios recitados ${ }^{31}$, cuya extensión permite afirmar que hay una tendencia a la brevedad: excepto Cab. 1326-32, que tiene siete versos y que presenta, además, la particularidad de ser el único dialogado -Morcillero-corifeo (el resto de las introducciones dialogadas en las comedias de Aristófanes son entre personajes)-, todos los demás son anuncios de dos versos (así dos de ellos, Ac. 1069 s. y Lis. 1072 s.), de tres (Ac. 238-40, Avisp. 1532-4, Aves 1196-8 y Tesm. 571-3, cuatro en total) o de cuatro (los dos de Lis. 1082-5 y As. 500-3). Otra tendencia clara se observa desde el punto de vista métrico, la de que el anuncio no posea un ritmo exclusivo para él; el único caso en el que esta tendencia se rompe lo hallamos entre las introducciones que acaban de ser estudiadas, Lis. 1072-3, dos tetrámetros anapésticos que siguen a un stasimon de ritmo fundamentalmente trocaico y dan paso a su vez a una escena yámbica. En cuanto a los metros empleados, lo más destacable es la variedad de los mismos, teniendo en cuenta que hay cinco diferentes en tan sólo nueve anuncios: a los registrados ahora (trímetros yámbicos, que encontramos en tres de ellos, Ac. 1069-70, Aves 1196-8 y Lis. 1082-5; tetrámetros yámbicos, en dos, Tesm. 571-3 y As. 500-3; y anapésticos, en otros dos, Cab. 1326-32 y Lis. 1072-3) hay que su-

31 Para el caso de los arquiloqueos de Avisp. 1532 ss., cf. los argumentos de L. E. Rossi («Mimica e danza sulla scena comica greca. A proposito del finale delle Vespe e di altri passi aristofanei», $R C C M 20,1978$, pp. 1149-1170, 1150-1151) a favor de una ejecución recitativa. Según el autor, la serie estíquica de arquiloqueos a la que pertenece el anuncio (1528-37) son versos recitados en $\pi \alpha \rho \alpha \kappa \alpha \tau \alpha \lambda o \gamma \eta$ por el corifeo sin acompañamiento de danza, y los arquiloqueos de la parte antistrófica precedente $(1518-22=1523-7)$ son cantados por el coro mientras baila. Zimmermann (Untersuchungen zur Form und dramatischen Technik der Aristophanischen Komödien. Die anderen lyrischen Partien II, Königstein/Ts., 1985, p. 84) estima probable la propuesta de Rossi. Nosotros la seguimos. Por su parte, van Daele los considera líricos, no haciendo así ninguna diferencia entre los arquiloqueos de un conjunto y otro. 
mar los tetrámetros trocaicos de Ac. 238-40 y los arquiloqueos de Avisp. 1532-4 (¡ninguno! de los cuatro anuncios de estas comedias tempranas tiene el mismo metro).

El contenido también merece una mención. Podemos afirmar que todos los anuncios en mayor o menor medida hacen algo más que constatar una entrada (ya se ha dicho que en el caso de Aves el contacto es sólo aural), añadiendo alguna breve información acerca de la misma o del personaje que la efectúa, o bien incluyendo la reacción del corifeo (en dos ocasiones pide silencio a sus compañeros, Ac. 238 y Tesm. 573). Por su contenido típico hemos destacado anteriormente Tesm. 571-3, y lo mismo cabe hacer con Ac. 1069-70, una parodia también del anuncio del mensajero trágico (se repiten en ambos los motivos de la prisa con la que el mensajero se aproxima y el de que trae noticias, a los que se suma, en $A c .1069$ s., el motivo de su gesto sombrío como presagio de malas nuevas ${ }^{32}$ ). Cab. 1326 ss., en cambio, sobresalía en el primer grupo de anuncios por su contenido «atípico» y lo hace igualmente una vez analizado el segundo; su tono solemne con tintes religio$\operatorname{sos}^{33}$ y las expresiones descriptivas que encierra -algunas muy gráficas y cargadas de valor simbólico, como los detalles a modo de acotaciones escénicas acerca de la apariencia externa de Pueblo (1331-2), reflejo del orden establecido al final de la comedia (Pueblo recupera su antigua soberanía)- lo separan de todos los demás. No es infrecuente, por otra parte, que los anuncios creen una cierta tensión dramática en torno a la entrada. Ya nos hemos referido a cómo la tragedia emplea con este propósito los motivos presentes en los dos anuncios de mensajero, haciendo lo mismo la comedia al tomarlos prestados. Pues bien, igual fin tienen el contacto aural previo a la entrada (el corifeo oye la petición de silencio formulada por Diceópolis desde dentro,

32 Sobre este motivo, Rau, Paratragodia 137-8. Ac. 1069-70 comienza además con el

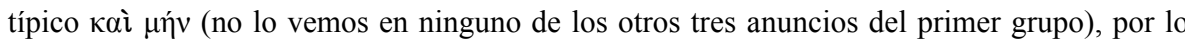
que resulta más estereotipado.

33 H. Kleinknecht, «Die Epiphanie des Demos in Aristophanes' Rittern», Hermes 77, 1939, pp. 58-65 (= Newiger, Aristophanes 144-54), sostiene que la entrada de Pueblo investido de nuevo de su poder soberano es una escena de epifanía configurada según el modelo cultual; una conclusión a la que llega después de analizar minuciosamente el vocabulario del anuncio y del resto de la esticomitia en la que éste se inserta. Véanse las matizaciones hechas por M. Landfester, Die Ritter des Aristophanes, Amsterdam 1967, pp. 92-97, a dicho análisis y a la interpretación del mismo. 
Ac. 238, y Morcillero, el ruido de los Propileos al abrirse, Cab. 1326; en Aves 1198 se percibe el ruido del batir de las alas del dios infiltrado) y la alusión explícita a la inmediatez de la misma (Cab. 1326) o a la cercanía del personaje (Aves 1197), recursos los tres con los que se crea la expectativa de la aparición de este último; la demora de la entrada (la expectativa de la llegada de Pueblo a escena se ve frustrada por un instante con la aparición de la antigua Atenas, Cab. 1327; Pueblo aparece en 1331) o del contacto verbal con el recién llegado (el coro se «retira» ante la entrada de Diceópolis, $A c$. 239-40, cuando se esperaba que se lanzara sobre él, lo que hace más tarde, 280) son fuente también de tensión dramática. En el caso de la entrada de Iris (Aves 1196), el anuncio no desvela la identidad de la mensajera de los dioses, lo cual, a pesar de que el corifeo no se pregunta por la misma ni hace ninguna conjetura al respecto, despierta el interés por saber de quién se trata $\mathrm{y}$, en consecuencia, por la propia entrada.

Por último, las introducciones en boca del corifeo constituyen un buen ejemplo de esa variedad de registros lingüísticos tan típica de la comedia: así, junto a los solemnes anapestos de Cab. 1326 ss., la poesía de Aves 1196 ss., la paratragedia de los dos anuncios de mensajero - Ac. 1069-70 y Tesm.

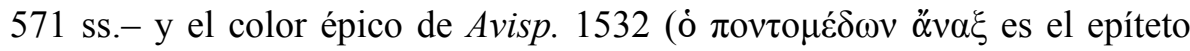
que se le atribuye a Cárcino al entrar en escena) se encuentra el estilo prosaico de Ac. 238-40, de As. 500-3 y el de los dos anuncios de Lis. 1072-3 y 1082-5, menos «neutro» que el de los anteriores por las alusiones sexuales, más o menos explícitas, que ambos contienen (el corifeo se percata de la erección de los embajadores atenienses y espartanos); alusiones, por otra

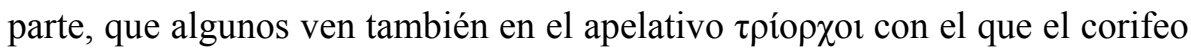
de Avisp. (1534) se refiere a los tres hijos de Cárcio ${ }^{34}$.

Pasemos ahora a ocuparnos de la segunda cuestión: las diferencias entre un conjunto de anuncios y otro. El lugar en el que éstos aparecen y el contacto posterior del coro con la skene constituyen, como hemos adelantado, tales

34 Tpíopұos es el nombre de una especie de gavilán (D'A. W. Thompson, A Glossary of

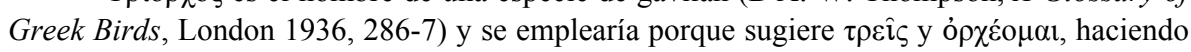
alusión al número y a la profesión de los Carcinitas, los «tres danzarines»; así D. M. MacDowell, Wasps, Oxford 1971, ad loc. En cambio, J. Henderson, The Maculate Muse, N. Y.-Oxford $1991^{2}$ (New Haven-London $\left.1975^{1}\right), 21$ y 125, señala que la palabra significa también «de tres testículos (ö $\rho \chi \varepsilon 1 \varsigma) »$, apuntando, por tanto, a un juego con reminiscencias sexuales, lo que MacDowell no hace. 
diferencias.

El análisis ha puesto de manifiesto que todos los anuncios del segundo grupo excepto uno (Lis. 1082-5) sirven de transición de un elemento coral o de una escena en versos largos (de la que el coro no permanece del todo al margen) a una escena yámbica, aminorando el paso de una unidad a otra distinta; ha revelado, igualmente, una cierta tendencia del anuncio a aparecer en el recitado en trímetros: así, Aves 1196-8 y Lis. 1082-5 se encuentran en sendas escenas yámbicas. Respecto a la actuación del corifeo tras el anuncio, hemos visto que, excepto en Aves, interviene en un diálogo con el recién llegado. En el caso de las obras estudiadas ahora, estos anuncios y diálogos insertados en el recitado yámbico hay que relacionarlos con el hecho de que el corifeo, a partir de Aves, habla más a menudo -lo que no significa mucho - en esta parte estructural de la comedia; las escenas yámbicas pertenecen propiamente a los personajes de la skene, y el papel que le corresponde al coro durante las mismas es el de mero observador. Su participación (a través del corifeo) en estas escenas es una forma, aunque tímida, de integrar al coro en la acción dramática durante toda la obra ${ }^{35}$.

Bien distinto es el panorama que ofrecen las introducciones del primer grupo. Sólo una de ellas aparece en un lugar de transición; se trata de $A c$. 238-40, la cual cierra el pequeño conjunto de versos, 234-40, que sigue a la sizigía ejecutada por el coro al irrumpir en la orquestra, 204-33, dando paso a la escena yámbica siguiente, 241-79 (cf. As. 500-3, cierre de la epiparodos). Y sólo una también forma parte de una escena yámbica (Ac. $1069 \mathrm{~s}$.); las demás, Cab. 1326-32 y Avisp. 1532-4, se encuentran, respectivamente, en una escena en versos largos (cf. Tesm. 571-3) y en la exodos. Por último, el corifeo interviene tras el anuncio en Cab. 1333-4 y Avisp. 1535-7, pero en ninguno de los dos casos lo hace dentro del recitado en trímetros y tampoco en ninguno de ellos su intervención da pie a un diálogo: Cab. 1333-4 es el ceremonioso saludo que el corifeo brinda a Pueblo, concluyendo así la esticomitia en tetrámetros anapésticos a la que pertenece el anuncio y, al mismo tiempo, la propia participación del coro en la comedia; en cuanto a Avisp. 1535-7, son los versos finales de la obra, en los que el corifeo sugiere a los

35 Gelzer, «Aristophanes», p. 1521: «Der Koryphaios spricht erst von den Vögeln an häufiger auch in Trimeterszenen, was mit der aktiveren Einbeziehung des Chors in die Handlung auch in zweiten Teil zusammenhängt».

EMERITA. Revista de Lingüística y Filología Clásica (EM) - LXV 2, 1997, pp. 287-302 
hijos de Cárcino, presentes en escena cuando llega su padre y personae mutae como él ${ }^{36}$, encabezar bailando la marcha final de salida.

En fin, sin olvidar la importancia de las diferencias que acabamos de apuntar, creemos que son más las características que unen a ambos grupos de anuncios que las que los separan. De los puntos en común señalados anteriormente recordamos para terminar los que nos parecen más destacables. En primer lugar, claro está, el reducido número de anuncios en boca del coro. En segundo lugar, el valor dramático que éstos adquieren en manos del poeta cómico: a pesar de su brevedad, no son en general elementos que sirvan de meros enlaces formales de una escena con otra; queremos resaltar, especialmente, la manera en que Aristófanes aprovecha en varios de ellos la posibilidad que brinda el anuncio de incrementar la expectación que crea por sí misma toda nueva entrada. El uso de la lengua cobra también relieve, destacando la variedad de estilos, un rasgo distintivo del «estilo aristofánico», capaz de pasar sin ningún esfuerzo de un nivel a otro. Por último, ciertos anuncios del corifeo son una muestra más de parodia de elementos formales típicos de la tragedia, como también lo es el diálogo que la corifeo de Tesm. mantiene con Clístenes tras anunciar la entrada de éste. Se trata, respectivamente, de la parodia del anuncio y del diálogo de entrada típicos de la intervención del mensajero en la tragedia.

IDOIA MAMOLAR SÁNCHEZ

36 El hecho de que Cárcino y sus hijos sean personajes mudos hace imposible, claro está, el diálogo. Por otra parte, y a diferencia de lo que sucede en el caso de Cab., lo único que podemos asegurar con certeza en éste es que el corifeo se dirige a personajes ya presentes en escena. En efecto, la alusión a la danza convierte a los Carcinitas en los destinatarios claros de sus palabras, y quizá a Filocleón, el otro personaje en escena, quien probablemente también bailaba poniendo el contrapunto cómico a la actuación experimentada de los tres hermanos; respecto a Cárcino, ya que nada se dice sobre si el motivo de su entrada es la mera contemplación del espectáculo coreográfico de sus hijos o su participación en él (cf. MacDowell, ad 1516-37), no es posible determinar con exactitud si está incluido o no entre los destinatarios de la sugerencia del corifeo. 